\title{
IDENTIFICATION OF BATES STOCHASTIC VOLATILITY MODEL BY USING NON-CENTRAL CHI-SQUARE RANDOM GENERATION METHOD
}

\author{
ShinIchi AIHARA, Arunabha BAGCH and Saikat SAHA \\ Tokyo University of Science,Suwa, 5000-1 Toyohira Chino,Nagano,Japan \\ FELab and Department of Applied Mathematics, University of Twente, Enschede, The Netherlands \\ Department of Electrical Engineering, Linköping University, SE-58183 Linköping, Sweden
}

\begin{abstract}
We study the identification problem for Bates stochastic volatility model, which is widely used as the model of a stock in finance. By using the exact simulation method, a particle filter for estimating stochastic volatility and its systems parameters is constructed. Simulation studies for checking the feasibility of the developed scheme are demonstrated.
\end{abstract}

Index Terms - Nonlinear filter, Particle filter, Stochastic volatility, Parameter estimation,Chi-square distribution

\section{INTRODUCTION}

In this paper, we estimate stochastic volatility and unknown system parameters in general stochastic volatility models with jumps as proposed by Bates [4] as given below

$d S_{t}=\left(\mu_{S}+\lambda_{S} v_{t}\right) S_{t} d t+\sqrt{v_{t}} S_{t} d B_{t}+S_{t} d Z_{t}^{J}-\lambda m^{J} S_{t} d t$

$d v_{t}=\left\{\kappa\left(\theta-v_{t}\right)+\lambda_{v} v_{t}\right\} d t+\xi \sqrt{v_{t}} d Z_{t}$

where $B_{t}$ and $Z_{t}$ are standard Brownian motion processes with correlation $\rho, Z_{t}^{J}$ denotes the pure-jump process which contains two components: random-event times and random jump sizes, and is independent of $B_{t}$ and $Z_{t}$. Denoting the intensity of the jump event time as $\lambda$ and the mean relative jump size as $m^{J}$, and by applying Ito's formula to $y_{t}=\log S_{t} / S_{0}$, we have

$d y_{t}=\left(\mu_{S}-\lambda m^{J}+\left(\lambda_{s}-\frac{1}{2}\right) v_{t}\right) d t+\sqrt{v_{t}} d B_{t}+d q_{t}^{J}$,

where $q_{t}^{J}$ is a compound Poisson process with intensity $\lambda$ and Gaussian distribution of jump size,i.e., $N\left(\mu_{J}, \sigma_{J}^{2}\right)$. Introducing the new Brownian motion

$$
\tilde{Z}_{t}=\frac{1}{\sqrt{1-\rho^{2}}}\left(Z_{t}-\rho B_{t}\right),
$$

(1) becomes

$$
\begin{array}{r}
d v_{t}=\kappa\left(\theta-v_{t}\right) d t+\xi \sqrt{v_{t}} \sqrt{1-\rho^{2}} d \tilde{Z}_{t} \\
+\xi \rho\left(d y_{t}-\left(\mu_{S}-\lambda m^{J}-\left(\frac{1}{2}-\lambda_{S}\right) v_{t}\right) d t-d q_{t}^{J}\right) .
\end{array}
$$

This work is partially supported by MECSST of Japan under Grant-inAid for Research(c) 17560402.
The systems parameters $\mu_{S}, \kappa, \theta, \xi, \lambda_{S}$ and $\lambda_{v}$ need to be calibrated from the historical data.

Our first problem is to estimate volatility $v_{t}$ based on observed data $y_{t}$. This is the usual filtering problem in signal processing. But the traditional extended Kalman filtering technique does not work in this situation, because a) the model is highly nonlinear, b) model has jumps, c) observation noise contains the system state.

The increasingly popular particle filtering technique method works extremely well in this situation [1]. One common problem of using particle filter in general is to obtain an appropriate importance function. In our model we can actually sample from the optimal importance function which is in itself a remarkable fact $[2,3]$. One serious difficulty of using particle filtering is the generation of the systems particles. Discrete approximations may lead to negative samples. To circumvent this problem, the exact simulation method has been recently proposed in [5]. Using this algorithm we can now formulate the particle filter where the transition and optimal importance functions are given by non-central chi square density functions.

Estimating unknown parameters in stochastic volatility models is known to be very difficult. The MLE does contain the usual difficult problem of multiple local maxima. Even the outputs from EM algorithm do not converge well, because of the shape of the chi-square probability density.

Although the usual augmented state approach does not always behave properly as shown in [1], we use this augmented state approach, because the bounds for unknown parameters are easily set a-priori and hence the estimate of the degrees of freedom of the non-central chi-square probability is well controlled in the required region for fitting commodity data.

\section{EXACT PARTICLE FILTERING}

\subsection{Exact sampling}

In order to implement the particle filter, the original system is usually approximated to the discrete-time one by using the Euler method. This approximation easily causes bias from the original continuous system. For example, the discrete- 
time volatility process $v_{k}$ often becomes negative. To avoid this bias, we propose the exact sampling method which is developed by Broadie and Kaya [5] for simulating the Heston process. In particle filter we generate samples from the optimal importance function $p\left(v_{t_{2}} \mid v_{t_{1}}, y_{t_{2}}, y_{t_{1}}\right)$. Now we shall present the exact sampling procedure. For simplicity we consider the time interval $t_{1}<t_{2}$ and set the following assumption: At most one jump occurs in this time interval and we observe $y_{t_{2}}$ and $y_{t_{1}}$.

1. Exact sampling from $p\left(v_{t_{2}} \mid v_{t_{1}}, y_{t_{2}}, y_{t_{1}}\right)$

From (2), the volatility process $v_{t_{2}}$ is represented by

$$
\begin{aligned}
v_{t_{2}} & =\tilde{v}_{t_{1}}+\int_{t_{1}}^{t_{2}} \tilde{\kappa}\left(\tilde{\theta}-v_{s}\right) d s \\
+ & \int_{t_{1}}^{t_{2}} \xi \sqrt{v_{s}} \sqrt{1-\rho^{2}} d \tilde{Z}_{s},
\end{aligned}
$$

where

$$
\begin{aligned}
\tilde{v}_{t_{1}}= & v_{t_{1}}+\rho \xi\left\{y_{t_{2}}-y_{t_{1}}\right. \\
& \left.-\left(\mu_{S}-\lambda m^{J}\right)\left(t_{2}-t_{1}\right)-\Delta q_{t_{1}}^{i}\right\} \\
\tilde{\kappa}= & \kappa-\frac{\rho \xi}{2}+\xi\left(\rho \lambda_{S}-\lambda_{v}\right) \\
\tilde{\theta}= & \frac{\kappa \theta}{\tilde{\kappa}} . \\
\Delta q_{t_{1}}^{i}= & \text { jump sample from } q_{t_{1}}^{J} \text { for } t_{1}<t<t_{2} .
\end{aligned}
$$

Now assuming that $\tilde{v}_{t_{1}} \geq 0$, we find that the transition law of $v_{t_{2}}$ given $v_{t_{1}}, y_{t_{1}}$ and $y_{t_{2}}$ is expressed as the non-central chi-square random variable $\chi_{d}^{2}\left(\lambda_{\chi}\right)$ with $d$ degree of freedom and non-centrality parameter $\lambda_{\chi}$,

$$
\frac{\xi^{2}\left(1-\rho^{2}\right)\left(1-e^{-\tilde{\kappa}\left(t_{2}-t_{1}\right)}\right)}{4 \tilde{\kappa}} \chi_{d}^{2}\left(\lambda_{\chi}\right),
$$

where

and

$$
d=\frac{4 \tilde{\theta} \tilde{\kappa}}{\xi^{2}\left(1-\rho^{2}\right)}
$$

$$
\lambda_{\chi}=\frac{4 \tilde{\kappa} e^{-\tilde{\kappa}\left(t_{2}-t_{1}\right)}}{\xi^{2}\left(1-\rho^{2}\right)\left(1-e^{-\tilde{\kappa}\left(t_{2}-t_{1}\right)}\right)} \tilde{v}_{t_{1}} .
$$

Hence by using MATLAB code "ncx2rnd.m", we can get a sample $v_{t_{2}}$.

For the case that $\tilde{v}_{t_{1}}<0$, which may occur when $v_{t_{1}}$ is very small, we need to adjust above procedure as described in the next step.

2. $\tilde{v}_{t_{1}}<0$ case

We reconstruct the data for $t_{1}<\tau_{1} \leq t_{2}$ such that

$$
\begin{aligned}
\Delta y & \left(\tau_{1}-t_{1}\right) \\
= & \frac{\rho \xi\left\{y_{t_{2}}-y_{t_{1}}-\left(\mu_{S}-\lambda m^{J}\right)\left(t_{2}-t_{1}\right)-\Delta q_{t_{1}}^{j}\right\}}{t_{2}-t_{1}} \\
& \times\left(\tau_{1}-t_{1}\right)
\end{aligned}
$$

and $\tau_{1}$ satisfies

$$
v_{t_{1}}+\Delta y\left(\tau_{1}-t_{1}\right) \geq 0 .
$$

where it is always possible to find $\tau_{1}$, because

$$
\lim _{\tau_{1} \rightarrow 0}\left\{v_{t_{1}}+\Delta y\left(\tau_{1}-t_{1}\right)\right\}=v_{t_{1}}>0 .
$$

By using the step 1., we obtain $v_{\tau_{1}}>0$. Now we check whether $v_{\tau_{1}}+\Delta y\left(t_{2}-\tau_{1}\right)$ is non-negative or not. If this is non-negative, we repeat the step 1. again and obtain $v_{t_{2}}>0$.If not we need to find $\tau_{2}$ :

$$
v_{\tau_{1}}+\Delta y\left(\tau_{2}-\tau_{1}\right) \geq 0 .
$$

Repeat above procedure, we finally obtain $v_{t_{2}}>0$. For $\tilde{\tilde{v}}_{t_{2}}<0$ case, we should use the same procedure mentioned here.

\subsection{Construction of probability density function}

If we use the Euler scheme, the generated sample becomes conditionally Gaussian. However in the exact sampling scheme, the processes generated are governed by the noncentral chi-square distribution. Although the explicit function form of this distribution is not possible, we can numerically evaluate the pdf by using the MATLAB code, "ncx2pdf.m".

- $p\left(v_{t_{2}} \mid v_{t_{1}}, y_{t_{2}}, y_{t_{1}}\right)$ form

Noting that the jump size $U_{\text {. }}^{s}$ is Gaussian with mean $\mu_{J}$ and variance $\sigma_{J}^{2}$, we have

$$
\begin{aligned}
& p\left(v_{t_{2}} \mid v_{t_{1}}, y_{t_{2}}, y_{t_{1}}\right) \\
& \times \operatorname{pdf} \text { of }\left\{\frac{\xi^{2}\left(1-\rho^{2}\right)\left(1-e^{-\tilde{\kappa}\left(t_{2}-t_{1}\right)}\right)}{4 \tilde{\kappa}} \chi_{d}^{2}\left(\tilde{\lambda}_{\chi}\right)\right\} \\
& +e^{-\lambda\left(t_{2}-t_{1}\right)} \lambda\left(t_{2}-t_{1}\right) \\
& \quad \times \int_{-\infty}^{\infty} \operatorname{pdf} \text { of }\left\{\frac{\xi^{2}\left(1-\rho^{2}\right)\left(1-e^{-\tilde{\kappa}\left(t_{2}-t_{1}\right)}\right)}{4 \tilde{\kappa}}\right. \\
& \left.\quad \times \chi_{d}^{2}\left(\tilde{\lambda}_{\chi}-\frac{4 \tilde{\kappa} e^{-\tilde{\kappa}\left(t_{2}-t_{1}\right)} \rho}{\xi\left(1-\rho^{2}\right)\left(1-e^{-\tilde{\kappa}\left(t_{2}-t_{1}\right)}\right)} U^{s}\right)\right\} \\
& \times \frac{1}{\sqrt{2 \pi \sigma_{J}^{2}}} \exp \left(-\frac{\left(U^{s}-\mu_{J}\right)^{2}}{2 \sigma_{J}^{2}}\right) d U^{s}
\end{aligned}
$$

where

$$
\begin{array}{r}
\tilde{\lambda}_{\chi}=\frac{4 \tilde{\kappa} e^{-\tilde{\kappa}\left(t_{2}-t_{1}\right)}}{\xi^{2}\left(1-\rho^{2}\right)\left(1-e^{-\tilde{\kappa}\left(t_{2}-t_{1}\right)}\right)}\left\{v_{t_{1}}\right. \\
\left.+\rho \xi\left\{y_{t_{2}}-y_{t_{1}}-\left(\mu_{S}-\lambda m^{J}\right)\left(t_{2}-t_{1}\right)\right\}\right\}
\end{array}
$$

In (7), the first term implies that we have no jump and the second term is caused by the jump size $U^{s} \in$ $N\left(\mu^{J}, \sigma_{J}^{2}\right)$. Furthermore in the second term we need to calculate the Gaussian integral. We may use some numerical procedure to calculate this but the best choise is still an open problem. 
- $p\left(v_{t_{2}} \mid v_{t_{1}}, y_{t_{1}}\right)$ form

It follows from (5) that

$$
\begin{aligned}
& p\left(v_{t_{2}} \mid v_{t_{1}}, y_{t_{1}}\right) \\
& \quad=\operatorname{pdf} \text { of } \frac{\xi^{2}\left(1-e^{-\left(\kappa-\xi \lambda_{v}\right)\left(t_{2}-t_{1}\right)}\right)}{4\left(\kappa-\xi \lambda_{v}\right)} \chi_{d}^{2}\left(\lambda_{\chi}^{v}\right),
\end{aligned}
$$

where

$$
d=\frac{4 \theta \kappa}{\xi^{2}}
$$

and

$$
\lambda_{\chi}^{v}=\frac{4\left(\kappa-\xi \lambda_{v}\right) e^{-\left(\kappa-\xi \lambda_{v}\right)\left(t_{2}-t_{1}\right)}}{\xi^{2}\left(1-e^{-\left(\kappa-\xi \lambda_{v}\right)\left(t_{2}-t_{1}\right)}\right)} v_{t_{1}}
$$

- $p\left(y_{t_{2}} \mid y_{t_{1}}, \int_{t_{1}}^{t_{2}} v_{s} d s\right)$ form In this case, we easily get

$$
\begin{aligned}
p\left(y_{t_{2}} \mid y_{t_{1}}, \int_{t_{1}}^{t_{2}} v_{s} d s\right)=\frac{1-e^{-\lambda\left(t_{2}-t_{1}\right)} \lambda\left(t_{2}-t_{1}\right)}{\sqrt{2 \pi\left(1-\rho^{2}\right) \int_{t_{1}}^{t_{2}} v_{s} d s}} \\
\times \exp \left[-\frac{1}{2\left(1-\rho^{2}\right) \int_{t_{1}}^{t_{2}} v_{s} d s}\left\{y_{t_{2}}-\left(y_{t_{1}}\right.\right.\right. \\
+\left(\mu_{S}-\lambda m^{J}-\frac{\kappa \theta \rho}{\xi}\right)\left(t_{2}-t_{1}\right) \\
\left.\left.\left.+\frac{\rho}{\xi}\left(v_{t_{2}}-v_{t_{1}}\right)\right)\right\}^{2}\right] \\
+\frac{e^{-\lambda\left(t_{2}-t_{1}\right)} \lambda\left(t_{2}-t_{1}\right)}{\sqrt{2 \pi\left(\left(1-\rho^{2}\right) \int_{t_{1}}^{t_{2}} v_{s} d s+\sigma_{J}^{2}\right)}} \\
\times \exp \left[-\frac{\kappa \rho}{2\left(\left(1-\rho^{2}\right) \int_{t_{1}}^{t_{2}} v_{s} d s+\sigma_{J}^{2}\right)}\left\{y_{t_{2}}^{t_{2}} d s\right.\right. \\
+\left(y_{t_{1}}+\left(\mu_{S}-\lambda m^{J}-\frac{\kappa \theta \rho}{\xi}\right)\left(t_{2}-t_{1}\right)\right. \\
\left.\left.\left.+\frac{\rho}{\xi}\left(v_{t_{2}}-v_{t_{1}}\right)\right)\right\}^{2}\right] \\
\quad-\left(\frac{\kappa \rho}{2}+\rho \lambda_{v}-\lambda_{S}\right) \int_{t_{1}}^{t_{2}} v_{s} d s+\mu^{J}
\end{aligned}
$$

\subsection{Exact particle filter algorithm}

Now we can perform the exact particle filter. The weight $w^{(i)}$ is given by the following recursive form:

$w_{t_{k}}^{(i)}=w_{t_{k-1}}^{(i)} \frac{p\left(y_{t_{k}} \mid y_{t_{k-1}}, \int_{t_{k-1}}^{t_{k}} v_{s}^{(i)} d s\right) p\left(v_{t_{k}}^{(i)} \mid v_{t_{k-1}}^{(i)}, y_{t_{k-1}}\right)}{p\left(v_{t_{k}}^{(i)} \mid v_{t_{k-1}}^{(i)}, y_{t_{k}}, y_{t_{k-1}}\right)}$.

The algorithm steps are:

- At each time $t_{k}$, using $y_{t_{k}}, y_{t_{k-1}}$, we generate particles $v_{t_{k}}^{(i)}$ from the algorithm (1) and calculate $p\left(v_{t_{k}}^{(i)} \mid v_{t_{k-1}}^{(i)}\right.$, $\left.y_{t_{k}}, y_{t_{k-1}}\right)$ given by (7).
- Using $v_{t_{k}}^{(i)}, v_{t_{k-1}}^{(i)}$, we calculate $p\left(v_{t_{k}}^{(i)} \mid v_{t_{k-1}}^{(i)}, y_{t_{k-1}}\right)$ given by (8).

- Using the above generated $\int_{t_{k-1}}^{t_{k}} v_{s}^{(i)} d s$ in the algorithm (2) and the observation data $y_{t_{k}}, y_{t_{k-1}}$, we calculate $p\left(y_{t_{k}} \mid y_{t_{k-1}}, \int_{t_{k-1}}^{t_{k}} v_{s}^{(i)} d s\right)$ from (9).

- Update the weight $w_{t_{k}}^{(i)}$ given by (10).

- In the above steps, we may use the resampling method, if needed.

\section{PARAMETER IDENTIFICATION}

Before constructing the parameter identification procedure, we will discuss about the noncentral chi-square probability density function. If the degrees of freedom $d$ defined by

$$
d=\frac{4 \kappa \theta}{\xi^{2}}
$$

takes its value greater than 2 , the log likelihood function is negative and convex and "zero" is not attainable. However if $d \leq 2$, the point "zero" is attainable and the function form of the log likelihood function becomes concave and its value becomes positive near zero point. Since in most practical applications in finance $d / 2<<1$, the log likelihood functional is not easy to be selected as the optimal cost. Furthermore if we use the EM-algorithm for finding the MLE for model parameters, in the maximization step we seek the next step optimal parameter value for fixing the state as the smoothed value with some fixed parameters and then the cost function tends to move to the infinity. This implies that we need the strict upper and lower bounds for unknown parameters for using EM-algorithm. That is, the value of the degrees of freedom should be fixed in proper region. However in practice, it is not possible to guess its value in advance. For avoiding these difficulties, we propose the usual filtering algorithm for identifying the system parameters.

\subsection{Parallel filtering}

For identifying the system parameters, we construct the parallel filtering algorithm for fixing the unknown parameter defined as

$$
\alpha=\left[\kappa \theta \xi \mu_{S} \rho \lambda \mu_{J} \sigma_{J}\right] .
$$

To perform the particle filter for $v_{k}$ with the fixed $\alpha$, we assume that

$$
\alpha \in U \text { (uniform distribution with known upper }
$$
and lower bounds).

To start our particle filter, we generate the initial pair particles

$$
\left(v_{0}^{(i)}, \alpha^{(i)}\right) \in \mathcal{N} \times U, i=1,2, \cdots, N .
$$


At each time step $t_{k}$ we generate $v_{k+1}^{(i)}$ with the fixed $\alpha^{(i)}$ from (5) and we can calculate (10). We then construct the estimates

$$
\hat{\alpha}_{t_{k}}=\sum_{i=1}^{N} \alpha^{(i)} w_{t_{k}}^{(i)} \text {, and } \hat{v}_{t_{k}}=\sum_{i=1}^{N} v_{t_{k}}^{(i)} w_{t_{k}}^{(i)} .
$$

\section{SIMULATION STUDIES}

We set the following parameters in Table 1 with their estimates: Here we set $d t=1 / 252$ and $M=2000$. The lower

Table 1. Model parameters and their estimates $(T=1)$

\begin{tabular}{c|ccccc}
\hline & $\kappa$ & $\theta$ & $\mu_{S}$ & $\rho$ & $\xi$ \\
\hline True & 0.864 & 1.100 & 0.060 & -0.150 & 2.100 \\
\hline Estimated & 0.830 & 1.037 & 0.057 & -0.147 & 2.059 \\
\hline \hline & $\lambda$ & $\mu^{J}$ & $\sigma^{J}$ & $\lambda_{v}$ & $\lambda_{S}$ \\
\hline True & 0.100 & -0.020 & 0.250 & 0.188 & 0.372 \\
\hline Estimated & 0.096 & -0.19 & 0.239 & 0.166 & 0.344 \\
\hline
\end{tabular}

and upper bounds for parameters are set as given in Table.2.

Table 2. Lower and upper bounds of model parameters

\begin{tabular}{c|ccccc}
\hline & $\kappa$ & $\theta$ & $\mu_{S}$ & $\rho$ & $\xi$ \\
\hline Upper bound & 0.993 & 1.265 & 0.069 & -0.113 & 2.415 \\
\hline Lower bound & 0.648 & 0.825 & 0.045 & -0.173 & 1.575 \\
\hline \hline & $\lambda$ & $\mu^{J}$ & $\sigma^{J}$ & $\lambda_{v}$ & $\lambda_{S}$ \\
\hline Upper bound & 0.115 & -0.015 & 0.287 & 0.216 & 0.428 \\
\hline Lower bound & 0.075 & -0.023 & 0.188 & 0.141 & 0.279 \\
\hline
\end{tabular}

Now we show the observation data $y_{t}$ and its log price .

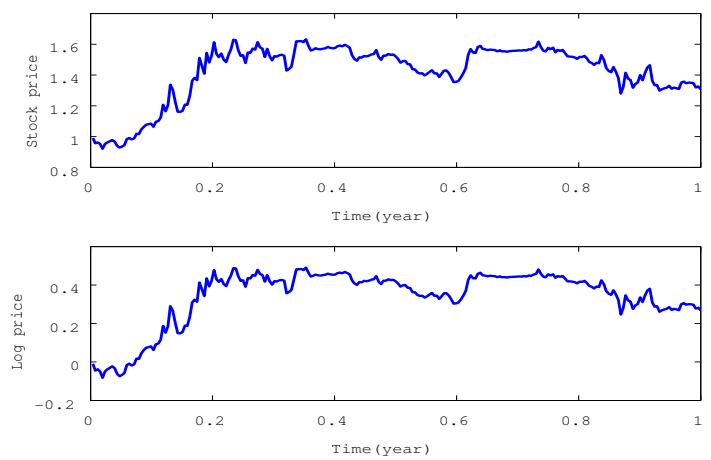

Fig. 1. Observation data $y_{t}$

In Fig.2, the true and estimated $v_{t}$ is demonstrated.

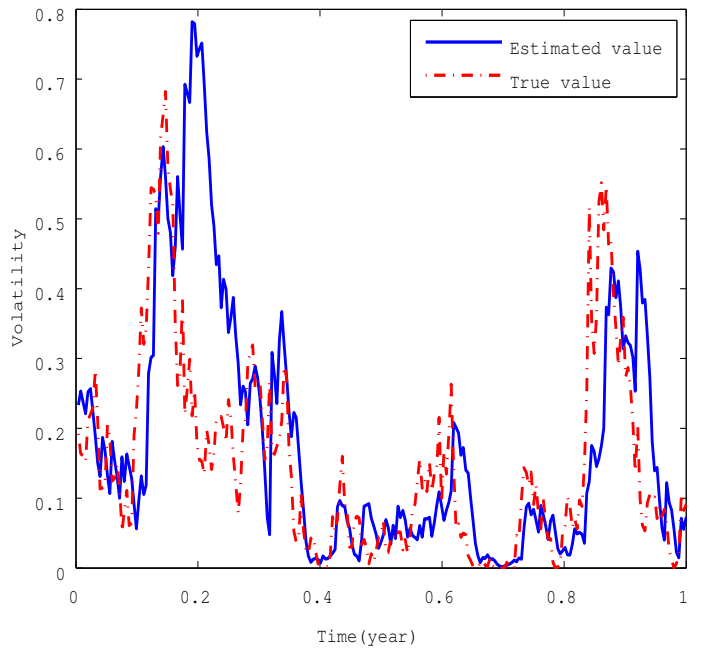

Fig. 2. True and estimated $v_{t}$

\section{CONCLUSIONS}

We developed a particle filter algorithm for estimating a stochastic volatility and its model parameters. It has been shown that the exact simulation technique used here works well even for the case that the degrees of freedom of a noncentral chi-square distribution is less that 2. Noting that the estimation procedure developed here is an on-line scheme, it is also possible to apply this scheme to a mean-variance hedging problem in finance.

\section{REFERENCES}

[1] S. Aihara, A. Bagchi, and S. Saha. Estimating volatility and model parameters of stochastic volatility models with jumps using particle filter. Proc. of 17th IFAC World Congress, 17, 2008.

[2] A. Doucet and S. Godsill and C. Andrieu On squential Monte Carlo sampling methods for Bayesian filtering. Statistic and Computing, 10, p.197-208,2000.

[3] RO. Cappé and E. Moulines and T .Rydén. Inference in Hidden Markov Models. Springer Science+Busimess Media Inc.NY, 2005.

[4] R. Cont and P. Tankov. Financial Modelling With Jump Processes. Chapman \& Hall/CRC, Boca Raton, 2004.

[5] M.Broadie and O. Kaya Exact simulation od Stochastic Volatility and Other Affine Jump Diffine Processes. Operations Research, 54, p.217-231,2006. 